\title{
Fracture resistance of extensive bulk-fill composite restorations after selective caries removal
}

Márcia Luciana Carregosa SANTANA(a) Lilian Fernanda Santos PAIVA(a) Vanda Sanderana Macedo CARNEIRO(b) Anderson Stevens Leônidas GOMES(c) Maximiliano Sérgio CENCI(d) (i) Andre Luis FARIA-E-SILVA(a)

(a) Universidade Federal de Sergipe - UFS, Graduate Program in Dentistry, Aracaju, SE, Brazil.

(b) Associação Caruaruense de Ensino Superior - Asces, Department of Dentistry, Caruaru, PE, Brazil.

(c) Universidade Federal de Pernambuco - UFPE, Department of Physics, Recife, PE, Brazil

(d) Universidade Federal de Pelotas - UFPel, School of Dentistry, Graduate Program in Dentistry, Pelotas, RS, Brazil.

Declaration of Interests: The authors certify that they have no commercial or associative interest that represents a conflict of interest in connection with the manuscript.

Corresponding Author:

André Luis Faria-e-Silva

E-mail: fariaesilva.andre@gmail.com

https://doi.org/10.1590/1807-3107bor-2020.vol34.011 1

Submitted: May 27. 2020

Accepted for publication: July 10, 2020

Last revision: July 23, 2020
Abstract: This study evaluated the effect of selective carious tissue removal on the fracture strength and failure mode of composite restorations in molars presenting only the buccal cusps. Deep cavities were prepared on the occlusal surface, and the lingual cusps were removed. Carious lesions in the middle of the pulpal wall were artificially induced with acetic acid $(\mathrm{pH}=4.5)$ for 35 days. The demineralized dentin was left intact or was completely removed prior to restoration with a bulk-fill composite $(n=10)$. Images of the specimens were obtained by optical coherence tomography (OCT) before and after the caries induction/removal. The mechanical resistance to fracture by axial compressive loading and the failure type and extension were determined. The pulpal wall/composite interface of the fractured specimens was analyzed by OCT. The data were analyzed for significance with t-tests $(\alpha=0.05)$. The deepest cavities and a more frequent occurrence of pulpal exposure were observed more often for non-selective carious tissue removal. The protocol of carious tissue removal did not affect the fracture strength $(p=0.554)$. An increased occurrence of catastrophic failures involving the roots was observed for non-selective carious tissue removal. Some occurrences of restoration displacement or cracks throughout the resin-dentin were observed only for the selective carious tissue approach. Selective carious tissue removal is a feasible approach to extensively damaged teeth since it reduced the occurrence of pulpal exposure and root fractures, without compromising the fracture strength.

Keywords: Dental Caries; Composite Resins; Dental Restoration Failure; Tomography, Optical Coherence.

\section{Introduction}

Caries disease results from dysbiosis associated with shifts of microbiota in dental biofilms, resulting in increased proportions of acid-producing and acid tolerating bacteria, and it is primarily driven by frequent sucrose consumption. .,2,3 $^{1}$ Therefore, reducing the $\mathrm{pH}$ of dental plaque promotes the demineralization of hard dental tissues, followed by degradation of the organic matrix, leading to cavitation. ${ }^{4}$ For a long time, treatment of cavitated caries lesions involved the complete removal of the demineralized dentin to avoid the progression of the 
lesions and to improve the biomechanical behavior of the restorations. ${ }^{5}$ However, using this approach increases the risk of pulp exposure and postoperative pulpal symptoms when the demineralized dentin is removed from the pulpal and axial walls of deep lesions. ${ }^{6,78,9}$

Nowadays, selective caries tissue removal and maintaining the soft demineralized dentin over the pulp is recommended for lesions reaching the inner third or quarter of the dentin. ${ }^{9}$ The presence of this outermost softened dentin that is left behind reduces the risk of pulp damage and arrests progression of the lesion. ${ }^{6,78,9,10,11,12,13,14,15}$ In contrast to the stepwise caries removal approach, the demineralized dentin is maintained under the restorative material because it might be detrimental to pulpal health to reenter the cavity to remove the carious tissue until "leathery" dentin is reached, ${ }^{912,15}$ Moreover, the re-entry procedure increases the treatment cost, time taken, and the discomfort to the patient. ${ }^{16}$

However, even if partial remineralization of the demineralized dentin left under the restoration were to occur ${ }_{1}^{17}$ the possibility of this remaining carious dentin causing an adverse effect such as reducing the mechanical properties and affecting the longevity of the restoration has not yet been elucidated. Indeed, several clinical trials have evaluated restorations placed after selective carious tissue removal for short follow-up periods. In addition to difficulties involved in evaluating restorations for a long period of time, it is barely possible to standardize the dimensions of teeth, cavities, and carious lesions in clinical studies. Furthermore, all of these factors can affect the biomechanical behavior of restorations.

In vitro studies assessing the biomechanical behavior of restorations placed after selective carious tissue removal have demonstrated conflicting results. Reduced fracture strength was observed when occlusal composite restorations were placed in decayed molars after the selective removal of carious tissue. ${ }^{18}$ In contrast, another in vitro study found no difference for selective and non-selective removal of carious tissue (artificially induced by an acid challenge) on the fracture strength of MOD restorations in premolars. ${ }^{19}$ Similar results were observed for bulk-fill composite restorations placed in MOD cavities prepared in molars with carious lesions in the pulpal wall, which were artificially induced by an acid challenge and a microcosm biofilm model. ${ }^{20}$ In addition to the differences in the cavity configurations, the various methods applied for caries induction also could explain the conflicting results. ${ }^{4,21,22}$

However, although deep carious lesions are usually observed in extensively damaged teeth, to the best of our knowledge, no previous study has evaluated the effect of selective carious removal in molars in the absence of cusps. Therefore, the present study aimed to evaluate the effect of selective carious removal on fracture resistance and the failure mode of composite restoration in molars presenting only the buccal cusps. The null hypothesis was that there would be no difference between the effect of nonselective and selective carious tissue removal on fracture resistance.

\section{Methodology}

In this ex vivo study, the independent variable was the protocol of removal (selective or non-selective) of carious tissue that was induced artificially in human molars by using an acid solution. The dependent variable analyzed was fracture resistance as the primary outcome. A sample size calculation was determined a priori by assuming a difference in fracture strength of $30 \%$ as significant, a test power of 0.80 , $\alpha$-error probability of 0.05 , for two experimental groups to be subjected to a t-test (SigmaStat v.3.5, Systat Software Inc., Chicago, USA). A variation coefficient of $22 \%$ was used based on a previous study, ${ }^{23}$ and ten specimens per experimental condition were required to meet the criteria above. The distribution of failure type and extension after the fracture resistance test, depth of the cavities, and the occurrence of pulpal exposure were also assessed.

Sound third molars with no cracks were selected, and based on the size and shape of their crowns, were allocated to the two experimental conditions in an endeavor to balance these factors. Digital periapical radiographs were taken to control the depth of the cavity preparations. For this purpose, a small segment of orthodontic steel wire was fixed $1 \mathrm{~mm}$ above the 
cementum-enamel junction on the buccal surface of the specimens, and the radiographic images were obtained using a $5 \mathrm{~mm}$ metal scale to allow the occlusal-pulpal distance to be measured. The distance between the steel wire and the pulp chamber roof was determined using the Image program (National Institutes of Health, Bethesda, USA), and a section was performed parallel to the occlusal surface at $1 \mathrm{~mm}$ above the pulp chamber roof distance, using a diamond disc (Extec, Enfield, USA) mounted in a cutting machine (Isomet Buehler, Lake Bluff, USA). A second section was performed perpendicular to the pulpal floor, maintaining only the buccal cusps. Further digital periapical radiographs were taken to ensure that the thickness of the remaining dentin was about $1 \mathrm{~mm}$ (Figure 1).

Before performing the caries induction protocol, the specimens were removed from the acrylic resin cylinders and the wax was removed from the root surfaces. Carious lesions were artificially induced in the middle of the pulpal wall. For this purpose, it was previously isolated with wax, and a thin layer of adhesive (Adper Universal, 3M ESPE, St. Paul, USA) was applied to the buccal wall and the peripheral regions of the pulpal wall, and light-cured for 20 seconds. The external surfaces of the specimens were covered with nail polish and the root apexes were closed with composite resin

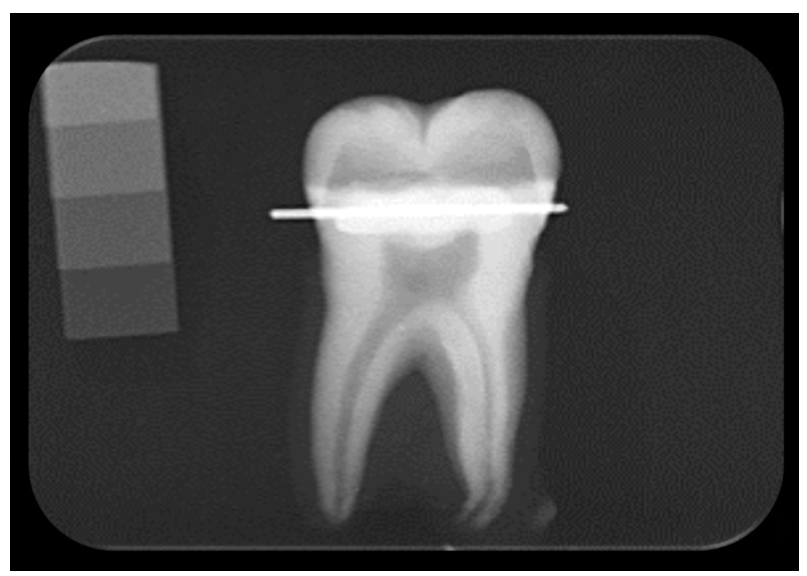

Figure 1. Periapical radiography used to control the depth of cavity preparation. Note the orthodontic steel wire (the more radiolucent object) helping to determine approximately $1 \mathrm{~mm}$ of remaining dentin between the occlusal surface and the pulp chamber roof.
(Filtek Z350, 3M-ESPE, St. Paul, USA). After this, the specimens were stored in a demineralizing solution (acetic acid $0.05 \mathrm{M}, \mathrm{pH}=4.5$ ) for 35 days. This time was defined in a preliminary study. ${ }^{21}$ The demineralizing solution was replaced daily and the $\mathrm{pH}$ of the solution was monitored.

No further procedure was performed for specimens allocated to the selective carious tissue removal protocol. An excavator was used to remove the demineralized softened dentin from the pulpal wall of the specimens allocated to the non-selective caries tissue removal protocol. The removal procedure was performed until only hard and dry dentin remained. Images of the specimens were obtained by using an optical coherence tomography (OCT) system (Callisto, Thorlabs Inc., Newton, USA). The system was operated in the spectral domain using a superluminescent diode light source at a central wavelength of $930 \mathrm{~nm}$, spectral bandwidth of $100 \mathrm{~nm}$, and maximum output power of $3 \mathrm{~mW}$, an axial resolution of $7 / 5.3 \mu \mathrm{m}$ (air/water), a lateral resolution of $8 \mu \mathrm{m}$, maximum imaging depth of $1.6 \mathrm{~mm}$, and axial scan rate of $1.2 \mathrm{kHz}$, capturing two frames per second with a sensitivity of $105 \mathrm{~dB} .^{24}$ The root surfaces were covered with melted wax and the specimens were inserted into acrylic resin cylinders (maintaining exposure of the coronal portion) to facilitate the scanning procedures. Two-dimensional OCT images were acquired perpendicular to the occlusal surface with buccal-lingual cross-sections with a $6 \mathrm{~mm}$ transverse scan. This whole system was controlled by a program that was developed based on the Labview computational tool (National Instruments, Austin, USA).

Afterward, the enamel was etched with 37\% phosphoric acid for 30 seconds, rinsed, and air-dried. The adhesive system (Adper Universal) was applied and light-cured for $20 \mathrm{~s}$, and the cavities were restored using a bulk-fill composite resin (Aura Bulk-Fill, SDI, Victoria, Australia) in a single increment directly placed on the cavity. The composite light-activation was performed with two $20 \mathrm{~s}$ light-curing procedures using an LED tip positioned over the occlusal and lingual faces. An LED-based unit Radii Cal (irradiance $\approx 1,000 \mathrm{~mW} / \mathrm{cm}^{2}$; SDI, Victoria, Australia) was used for all of the light-curing procedures. No pulp capping material was used under the composite despite its 
clinical indication in deep cavities, mainly in the presence of pulpal exposure. This approach was chosen to eliminate the effect of the mechanical properties of pulp capping material on the fracture resistance of the composite restoration.

Before the fracture resistance test, the specimen roots were covered with a polyether impression material (Impregum, 3M ESPE, St. Paul, USA) to simulate the periodontal ligament and they were then inserted into the acrylic resin cylinders. The fracture resistance was assessed by applying an axial compressive load on the occlusal surfaces of the specimens by means of an $8 \mathrm{~mm}$ stainless-steel sphere, at a crosshead speed of $0.5 \mathrm{~mm} / \mathrm{min}$, in a universal testing machine (MTS Landmark 370.10, MTSSystems Corporation, Eden Prairie, USA) until the specimen fractured. The maximum load required to cause specimens to fracture was recorded in Newtons.

The failure type and extension of each specimen were assigned to one of the following categories: a) fractures involving a small portion of the coronal tooth structure; b) fractures involving a small portion of the coronal tooth structure and cohesive failure of the restoration; c) fractures involving the tooth structure, cohesive and/or adhesive failure of the restoration, with root involvement, that was deemed restorable; and d) severe root and crown fracture, cohesive and/or adhesive failure of the restoration, which was deemed non-restorable.

Except for the specimens presenting with type IV failure, the specimens were sectioned at the enamelcement junction to expose the pulp chamber and to allow for proper scanning of the pulpal wall/composite interface by OCT. The integrity of this interface was qualitatively analyzed. The data from the fracture resistance test presented a normal distribution and equal variance assessed by the Shapiro-Wilk $(p=0.462)$ and Levene tests $(p=0.544)$, respectively. Therefore, data analysis was performed with the t-test. The Chi-square test was not applied to the failure mode data, because over $25 \%$ of the expected values in the contingency table were lower than 5 , compromising the accuracy of the test. The significance level was set at $95 \%$ and the analysis was performed using the Sigma-Stat v.3.5 statistical software package (Systat Software Inc., Chicago, USA).

\section{Results}

Images captured by means of OCT demonstrated that shallow cavities had been obtained in the pulpal wall by immersing the specimens in acetic acid for 35 days (Figure 2A). However, the demineralization process produced soft dentin in the pulpal wall and deeper cavities formed after soft dentin removal with an excavator (non-selective carious tissue removal) than that observed for selective carious tissue removal (Figure 2B). Seven specimens allocated to the non-selective carious tissue removal group and three in the selective carious tissue removal group presented exposure of the pulp chamber (Figure 2C).

No significant effect of the carious tissue removal protocol on fracture strength $(p=0.554)$ was observed (Table 1). Moreover, similar values of fracture strength were observed for specimens presenting or not with micro-exposure of the pulpal chamber. As regards the failure mode (Table 2), an increased tendency to fractures involving the roots (type III and IV) was observed for non-selective carious tissue removal and in the presence of micro-exposure of the pulp. Figure 3 illustrates some specimens that developed non-restorable fractures (type IV). The pulpal wall/ composite interfaces of four fractured specimens for non-selective carious tissue removal were analyzed (the others experienced fracture type IV) and they were all intact. For selective carious tissue removal, five interfaces were intact, two demonstrated some resin displacement, and cracks throughout the resindentin were observed in one specimen. Figure 4 shows representative images of the analyzed interfaces.

\section{Discussion}

Selective carious tissue removal has been advocated in the presence of deep cavities involving the inner third of the dentin to reduce the risk of pulp damage. .,8,9 $^{2}$ In spite of the strong evidence regarding the efficacy of using this conservative approach for maintaining pulp vitality, the biomechanical behavior of the restorations placed over the demineralized dentin continues to be a cause of concern, particularly when extensively damaged teeth are being treated. The rationale is that the presence of underlying dentin 


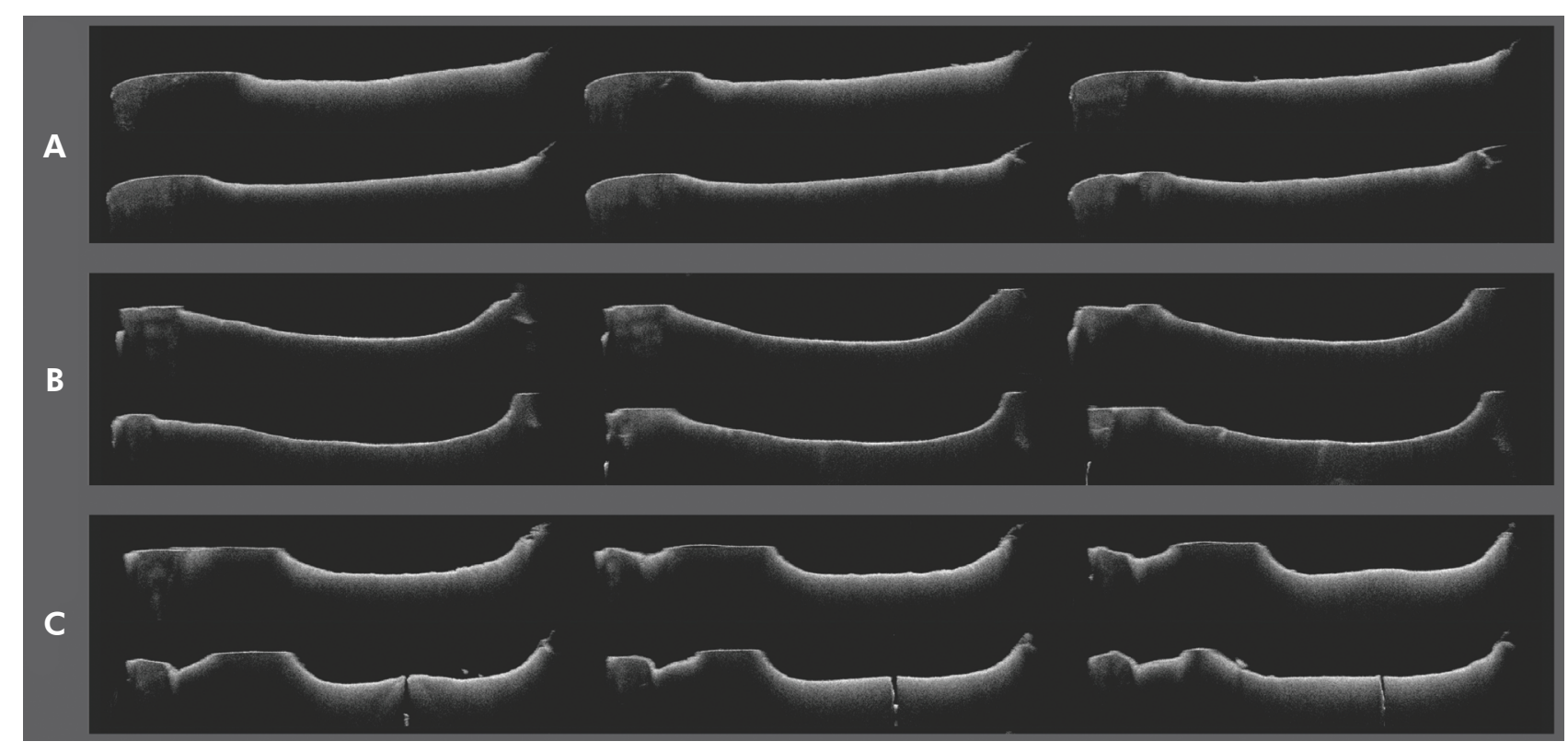

Figure 2. Sequential images obtained by OCT of a specimen allocated to the selective carious tissue removal protocol after caries induction $(A)$, and the non-selective carious tissue removal protocol after caries induction and softened dentin removal with an excavator (B and C). Note the pulp chamber exposure due to the deep cavity obtained in $\mathrm{C}$.

Table 1. Means \pm standard deviation $(n)$ of fracture strength according to the carious tissue removal protocol and the presence of micro-exposures of the pulpal chamber.

\begin{tabular}{lccc}
\hline \multirow{2}{*}{ Carious tissue removal protocol } & \multicolumn{2}{c}{ Micro-exposures of the pulpal chamber } & \multicolumn{2}{c}{ Pooled average } \\
\cline { 2 - 4 } & \multicolumn{1}{c}{ Present } & Absent & $(\mathrm{n}=10)$ \\
\hline Non-selective & $1,124.3 \pm 426.1(7)$ & $1,100.0 \pm 302.0(3)$ & $1,177.0 \pm 376.1$ \\
Selective & $1,290.0 \pm 728.6(3)$ & $1,224.3 \pm 525.1(7)$ & $1,244.0 \pm 550.2$ \\
\hline
\end{tabular}

Table 2. Failure mode according to the carious tissue removal protocol and the presence of micro-exposures of the pulpal chamber.

\begin{tabular}{|c|c|c|c|c|c|}
\hline \multirow{2}{*}{ Carious tissue removal protocol } & \multirow{2}{*}{ Micro-exposures of the pulpal chamber } & \multicolumn{4}{|c|}{ Failure mode } \\
\hline & & Type I & Type II & Type III & Type IV \\
\hline \multirow{3}{*}{ Non-selective } & Present & 0 & 1 & 1 & 5 \\
\hline & Absent & 0 & 2 & 0 & 1 \\
\hline & Total & 0 & 3 & 1 & 6 \\
\hline \multirow{3}{*}{ Selective } & Present & 0 & 1 & 0 & 2 \\
\hline & Absent & 0 & 6 & 1 & 0 \\
\hline & Total & 0 & 7 & 1 & 2 \\
\hline
\end{tabular}

(I) Fractures involving a small portion of the coronal tooth structure; (II) fractures involving a small portion of the coronal tooth structure and cohesive failure of the restoration; (III) fractures involving the tooth structure, cohesive, and/or adhesive failure of the restoration, with root involvement, that were deemed restorable; and (IV) severe root and crown fracture, cohesive and/or adhesive failure of the restoration, which were deemed non-restorable.

results in worse mechanical properties (such as the elastic modulus) than those observed for sound dentin, which might lead to the collapse of the restoration under occlusal loading. However, the findings of the present study demonstrated that both carious tissue removal protocols yielded similar fracture 
resistance of the composite restorations built-up in molars presenting only the buccal cusps. Therefore, the null hypothesis of the study was accepted.

Although it is difficult to mimic the features of natural caries lesions, several protocols of artificial carious lesion induction in dentin have been described in in vitro studies. ${ }^{4,19,20,21,22,23}$ The main advantage of artificially inducing dentinal lesions is to control the depth, location, and mineral loss of the lesions, thereby standardizing the specimens. Dentin demineralization can be achieved either by immersion of the specimens

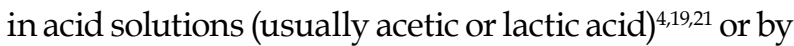

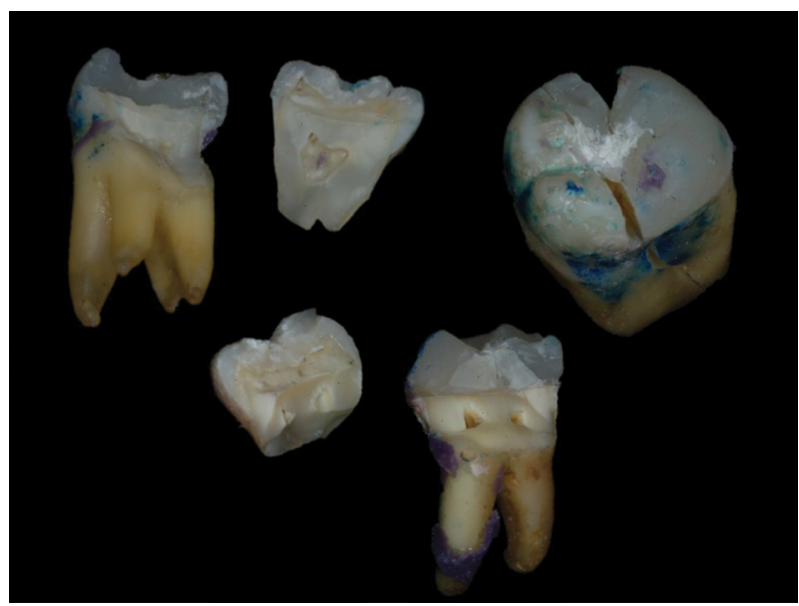

Figure 3. Illustrative images of specimens with severe root and crown fractures classified as non-restorable (Type IV). exposure to specific (Streptococcus mutans) ${ }^{22}$ and nonspecific biofilms..$^{20}$ In the present study, artificial carious lesions were induced in the middle of the pulpal wall with an acetic acid solution as described in a previous study. ${ }^{21}$ This protocol allowed the production of standardized lesions, appropriate for the experimental design of this study, although this protocol probably preserved the organic dentin matrix to a greater extent than for natural caries lesions. The protocol used here (acetic acid for 35 days) has been shown to result in values of lesion depth $(\approx 360 \mu \mathrm{m})$, mineral loss $(\approx 9.1 \mathrm{vol} \%$ $\times \mu \mathrm{m})$ and mineral loss ratio $(\approx 26.9 \mathrm{vol} \%)$ close to those observed for natural lesions $(\approx 626 \mu \mathrm{m}, \approx 12.4 \mathrm{vol} \% \times$ $\mu \mathrm{m}$ and $\approx 19.5 \mathrm{vol} \%$, respectively). ${ }^{21}$

Therefore, it was expected that approximately a third of the remaining dentin left in the pulpal wall $(1 \mathrm{~mm})$ after the cavity preparation would be demineralized. However, the occurrence of pulp exposure (mostly in the non-selective protocols) was observed for both carious tissue removal protocols, indicating that the radiographic images used to guide the depth of cavity preparations were not totally effective. A previous study showed that radiographic images could overestimate dentin wall thickness. ${ }^{25}$ In fact, the sequential images obtained by OCT allowed for the visualization of small exposures that had not been observed when using a single two-dimensional image provided by periapical radiography. The pulpal micro-exposures could act as stress-concentration areas favoring tooth fracture or

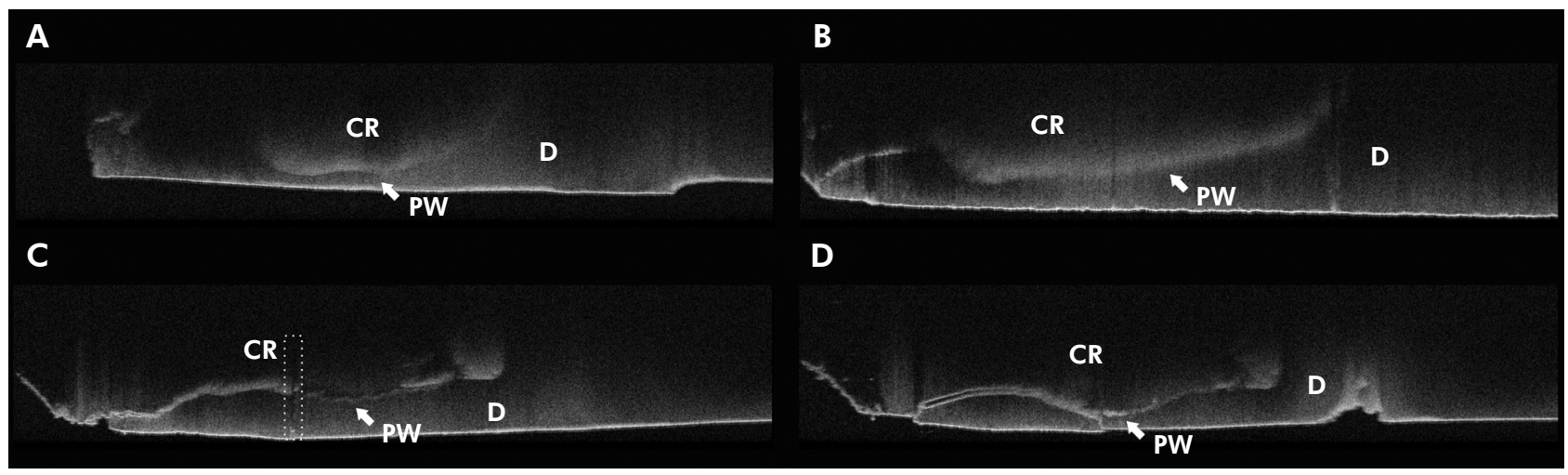

Figure 4. Representative images showing the interface between the bottom of the composite resin and the pulp wall of the cavity after the fracture resistance test. Intact interfaces for non-selective (A) and selective (B) carious tissue protocols. (C) Note the crack throughout the dentin and composite resin (inside the rectangle with dashed white lines) for a specimen with selective carious tissue removal. (D) Composite displacement in a specimen with selective carious tissue removal. CR - composite resin; D - dentin; and PW - pulpal wall. 
modifying the failure mode. Nevertheless, teeth that presented these occurrences were maintained in the study because the presence of micro-exposures did not affect the fracture resistance of the restorations. On the other hand, there was a tendency for more catastrophic failure modes in the presence of microexposures of the pulpal chamber.

Different from other previous in vitro studies assessing the effect of selective carious removal on fracture resistance, ${ }^{18-20}$ the present study removed the lingual cusps during cavity preparation and the restoration was retained mainly due to bonding to the remaining buccal wall. There is controversy about whether a direct restoration using composite can be indicated in extensively damaged teeth because the longevity of the restoration could be reduced. However, it is important to emphasize that selective carious tissue removal is a feasible approach to reduce the indications of endodontic treatment, and consequently, the cost of dental treatment. Therefore, this protocol favors low-income patients to maintain their teeth presenting with extensive carious lesions, but it is also necessary for the use of restorative procedures other than indirect restorations, which have a high cost. Thus, we chose to restore the molars using a direct restoration with a bulk-fill composite resin to avoid more expensive restorative protocols.

Indeed, removing the lingual cusps caused a reduction of 33\% in fracture resistance when compared with MOD restorations performed in molars with a similar composite. ${ }^{20}$ However, despite the lower fracture resistance values obtained due to the cavity configuration, the carious tissue removal protocol did not affect the fracture resistance in the present study, and these findings were in agreement with previous studies using artificially induced carious lesions. ${ }^{19,20}$ Besides the fracture resistance values, the extension of the failure is also important because more favorable failures allow for replacement of the restoration. Interestingly, the selective carious tissue removal protocol resulted in fewer failures involving the root than the non-selective approach. A possible explanation could be that the presence of more resilient dentin under the restoration helped to absorb the stress caused by compressive loading, and this reduced crack propagation throughout the tooth structure. ${ }^{26}$ In fact, analysis of the dentin/ composite interfaces of specimens not presenting catastrophic failures demonstrated the occurrence of resin displacement or crack formation when selective carious tissue removal was performed.

The findings of the present study add to the evidence supporting the use of the selective carious tissue removal approach to treat decayed teeth with deep cavities that reach the inner third of the dentin. This more conservative protocol reduced the occurrence of pulp exposure and root fracture, without compromising the fracture resistance of the restorations. However, these results must be carefully analyzed since artificial caries induction is unable to reproduce all features of natural lesions. Moreover, the static loading used to assess fracture resistance is poorly correlated with clinical situations when failure is due to the fatigue phenomenon caused by the subcritical dynamic (cyclic) load.

\section{Conclusions}

Selective carious tissue removal did not affect the fracture strength of molars presenting only the buccal cusps and restored with a bulk-fill composite when compared with the non-selective approach. Furthermore, selective carious tissue removal reduced the occurrence of pulp exposure and root fracture.

\section{Acknowledgments}

M.L.C.S is grateful to Capes for her scholarship. This study was partially supported by CAPES/ FAPITEC/SE \#11/2016, Brazil (protocol number: 121.034.052.192.113.000.000).

\section{References}

1. Sheiham A, James WP. Diet and dental caries: the pivotal role of free sugars reemphasized. J Dent Res. 2015 Oct;94(10):1341-7. https://doi.org/10.1177/0022034515590377 
Fracture resistance of extensive bulk-fill composite restorations after selective caries removal

2. Giacaman RA. Sugars and beyond. The role of sugars and the other nutrients and their potential impact on caries. Oral Dis. 2018 Oct;24(7):1185-97. https://doi.org/10.1111/odi.12778

3. Head D, A Devine D, Marsh PD. In silico modelling to differentiate the contribution of sugar frequency versus total amount in driving biofilm dysbiosis in dental caries. Sci Rep. 2017 Dec;7(1):17413. https://doi.org/10.1038/s41598-017-17660-z

4. Chien YC, Burwell AK, Saeki K, Fernandez-Martinez A, Pugach MK, Nonomura G, et al. Distinct decalcification process of dentin by different cariogenic organic acids: Kinetics, ultrastructure and mechanical properties. Arch Oral Biol. 2016 Mar;63:93-105. https://doi.org/10.1016/i.archoralbio.2015.10.001

5. Kidd EA. How 'clean' must a cavity be before restoration? Caries Res. 2004 May-Jun;38(3):305-13. https://doi.org/10.1159/000077770

6. Thompson V, Craig RG, Curro FA, Green WS, Ship JA. Treatment of deep carious lesions by complete excavation or partial removal: a critical review. J Am Dent Assoc. 2008 Jun;139(6):705-12. https://doi.org/10.14219/jada.archive.2008.0252

7. Manton D. Partial caries removal may have advantages but limited evidence on restoration survival. Evid Based Dent. 2013 Sep;14(3):74-75. https://doi.org/10.1038/sj.ebd.6400948

8. Schwendicke F, Meyer-Lueckel H, Dörfer C, Paris S. Failure of incompletely excavated teeth: a systematic review. J Dent. 2013 Jul;41(7):569-80. https://doi.org/10.1016/i.jdent.2013.05.004

9. Schwendicke F, Frencken JE, Bjørndal L, Maltz M, Manton DJ, Ricketts D, et al. Managing carious lesions: consensus recommendations on carious tissue removal. Adv Dent Res. 2016 May;28(2):58-67. https://doi.org/10.1177/0022034516639271

10. Oliveira EF, Carminatti G, Fontanella V, Maltz M. The monitoring of deep caries lesions after incomplete dentine caries removal: results after 14-18 months. Clin Oral Investig. 2006 Jun;10(2):134-9. https://doi.org/10.1007/s00784-006-0033-8

11. Maltz M, Oliveira EF, Fontanella V, Carminatti G. Deep caries lesions after incomplete dentine caries removal: 40-month follow-up study. Caries Res. 2007;41(6):493-6. https://doi.org/10.1159/000109349

12. Bjørndal L, Reit C, Bruun G, Markvart M, Kjaeldgaard M, Näsman P, et al. Treatment of deep caries lesions in adults: randomized clinical trials comparing stepwise vs. direct complete excavation, and direct pulp capping vs. partial pulpotomy. Eur J Oral Sci. 2010 Jun;118(3):290-7. https://doi.org/10.1111/j.1600-0722.2010.00731.x

13. Maltz M, Alves LS, Jardim JJ, Moura MS, Oliveira EF. Incomplete caries removal in deep lesions: a 10-year prospective study. Am J Dent. 2011 Aug;24(4):211-4.

14. Maltz M, Jardim JJ, Mestrinho HD, Yamaguti PM, Podestá K, Moura MS, et al. Partial removal of carious dentine: a multicenter randomized controlled trial and 18-month follow-up results. Caries Res. 2013;47(2):103-9. https://doi.org/10.1159/000344013

15. Hoefler $\mathrm{V}$, Nagaoka H, Miller CS. Long-term survival and vitality outcomes of permanent teeth following deep caries treatment with step-wise and partial-caries-removal: a systematic review. J Dent. 2016 Nov;54:25-32. https://doi.org/10.1016/i.jdent.2016.09.009

16. Schwendicke F, Stolpe M, Meyer-Lueckel H, Paris S, Dörfer CE. Cost-effectiveness of one- and two-step incomplete and complete excavations. J Dent Res. 2013 Oct;92(10):880-7. https://doi.org/10.1177/0022034513500792

17. Bertassoni LE, Habelitz S, Marshall SJ, Marshall GW. Mechanical recovery of dentin following remineralization in vitro: an indentation study. J Biomech. 2011 Jan;44(1):176-81. https://doi.org/10.1016/i.jbiomech.2010.09.005

18. Hevinga MA, Opdam NJ, Frencken JE, Truin GJ, Huysmans MC. Does incomplete caries removal reduce strength of restored teeth? J Dent Res. 2010 Nov;89(11):1270-5. https://doi.org/10.1177/0022034510377790

19. Schwendicke F, Kern M, Meyer-Lueckel H, Boels A, Doerfer C, Paris S. Fracture resistance and cuspal deflection of incompletely excavated teeth. J Dent. 2014 Feb;42(2):107-13. https://doi.org/10.1016/i.jdent.2013.12.003

20. Silva PF, Oliveira LR, Braga SS, Signori C, Armstrong SR, Soares CJ, et al. Effect of selective carious tissue removal on biomechanical behavior of class II bulk-fill dental composite restorations. Dent Mater. 2018 Sep;34(9):1289-98. https://doi.org/10.1016/i.dental.2018.05.014

21. Schwendicke F, Eggers K, Meyer-Lueckel H, Dörfer C, Kovalev A, Gorb S, et al. In vitro Induction of residual caries lesions in dentin: comparative mineral loss and nano-hardness analysis. Caries Res. 2015;49(3):259-65. https://doi.org/10.1159/000371897

22. Lenzi TL, Calvo AF, Tedesco TK, Ricci HA, Hebling J, Raggio DP. Effect of method of caries induction on aged resin-dentin bond of primary teeth. BMC Oral Health. 2015 Jul;15(1):79. https://doi.org/10.1186/s12903-015-0049-z

23. Kucukyilmaz E, Yasa B, Akcay M, Savas S, Kavrik F. Effects of pulp capping materials on fracture resistance of Class II composite restorations. Eur J Dent. 2015 Apr-Jun;9(2):218-23. https://doi.org/10.4103/1305-7456.156831

24. Mota CCO, Gueiros LA, Maia AM, Santos-Silva AR, Gomes AS, Alves FA, et al. Optical coherence tomography as an auxiliary tool for the screening of radiation-related caries. Photomed Laser Surg. 2013 Jul;31(7):301-6. https://doi.org/10.1089/pho.2012.3415

25. Souza EM, Bretas RT, Cenci MS, Maia-Filho EM, Bonetti-Filho I. Periapical radiographs overestimate root canal wall thickness during post space preparation. Int Endod J. 2008 Aug;41(8):658-63. https://doi.org/10.1111/i.1365-2591.2008.01410.x

26. Opdam NJ, Bronkhorst EM, Roeters JM, Loomans BA. Longevity and reasons for failure of sandwich and total-etch posterior composite resin restorations. J Adhes Dent. 2007 Oct;9(5):469-75. 